DESY $00-027$

ISSN $0418-9833$

February 2000

\title{
Search for Compositeness, Leptoquarks and Large Extra Dimensions in $e q$ Contact Interactions at HERA
}

\author{
H1 Collaboration
}

\begin{abstract}
The reaction $e^{+} p \rightarrow e^{+} X$ is studied with the H1 detector at HerA. The data cover momentum transfers $Q^{2}$ between $200 \mathrm{GeV}^{2}$ and $30,000 \mathrm{GeV}^{2}$ and correspond to an integrated luminosity of $35.6 \mathrm{pb}^{-1}$. The differential cross section $\mathrm{d} \sigma / \mathrm{d} Q^{2}$ is compared to the Standard Model expectation for neutral current scattering and analysed to search for $(\bar{e} e)(\bar{q} q)$ contact interactions. No evidence for new phenomena is observed. The results are used to set limits on scales within models of electron-quark compositeness, quark form factors and the exchange of virtual heavy leptoquarks. A search for gravitational effects mediated through the exchange of virtual gravitons which propagate into large extra dimensions is presented.
\end{abstract}


C. Adloff ${ }^{33}$, V. Andreev ${ }^{24}$, B. Andrieu ${ }^{27}$, V. Arkadov ${ }^{35}$, A. Astvatsatourov ${ }^{35}$, I. Ayyaz ${ }^{28}$, A. Babaev ${ }^{23}$, J. Bähr ${ }^{35}$, P. Baranov ${ }^{24}$, E. Barrelet ${ }^{28}$, W. Bartel ${ }^{10}$, U. Bassler $^{28}$, P. Bate $^{21}$, A. Beglarian ${ }^{34}$, O. Behnke ${ }^{10}$, C. Beier ${ }^{14}$, A. Belousov ${ }^{24}$, T. Benisch ${ }^{10}$, Ch. Berger ${ }^{1}$, G. Bernardi ${ }^{28}$, T. Berndt ${ }^{14}$, G. Bertrand-Coremans ${ }^{4}$, J.C. Bizot ${ }^{26}$, K. Borras ${ }^{7}$, V. Boudry ${ }^{27}$, W. Braunschweig ${ }^{1}$, V. Brisson ${ }^{26}$, H.-B. Bröker ${ }^{2}$, D.P. Brown ${ }^{21}$, W. Brückner ${ }^{12}$, P. Bruel ${ }^{27}$, D. Bruncko ${ }^{16}$, J. Bürger ${ }^{10}$, F.W. Büsser ${ }^{11}$, A. Bunyatyan ${ }^{12,34}$, H. Burkhardt ${ }^{14}$, A. Burrage ${ }^{18}$, G. Buschhorn ${ }^{25}$, A.J. Campbell ${ }^{10}$, J. Cao ${ }^{26}$, T. Carli ${ }^{25}$, S. Caron ${ }^{1}$, E. Chabert ${ }^{22}$, D. Clarke ${ }^{5}$, B. Clerbaux ${ }^{4}$, C. Collard ${ }^{4}$, J.G. Contreras ${ }^{7,41}$, J.A. Coughlan ${ }^{5}$, M.-C. Cousinou ${ }^{22}$, B.E. Cox ${ }^{21}$, G. Cozzika ${ }^{9}$, J. Cvach ${ }^{29}$, J.B. Dainton ${ }^{18}$, W.D. Dau ${ }^{15}$, K. Daum ${ }^{33,39}$, M. David ${ }^{9, \dagger}$, M. Davidsson ${ }^{20}$, B. Delcourt ${ }^{26}$, N. Delerue ${ }^{22}$, R. Demirchyan ${ }^{34}$, A. De Roeck ${ }^{10,43}$, E.A. De Wolf ${ }^{4}$, C. Diaconu ${ }^{22}$, P. Dixon ${ }^{19}$, V. Dodonov ${ }^{12}$, K.T. Donovan ${ }^{19}$, J.D. Dowell ${ }^{3}$,

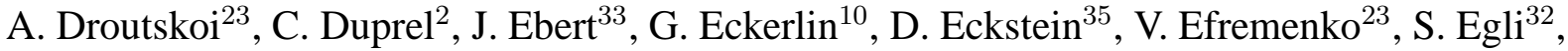
R. Eichler ${ }^{36}$, F. Eisele ${ }^{13}$, E. Eisenhandler ${ }^{19}$, M. Ellerbrock ${ }^{13}$, E. Elsen ${ }^{10}$, M. Erdmann ${ }^{10,40, e}$, W. Erdmann ${ }^{36}$, P.J.W. Faulkner ${ }^{3}$, L. Favart ${ }^{4}$, A. Fedotov ${ }^{23}$, R. Felst ${ }^{10}$, J. Ferencei ${ }^{10}$, F. Ferrarotto ${ }^{31}$, S. Ferron ${ }^{27}$, M. Fleischer ${ }^{10}$, G. Flügge ${ }^{2}$, A. Fomenko ${ }^{24}$, I. Foresti $^{37}$, J. Formánek ${ }^{30}$, J.M. Foster ${ }^{21}$, G. Franke ${ }^{10}$, E. Gabathuler ${ }^{18}$, K. Gabathuler ${ }^{32}$, J. Garvey ${ }^{3}$, J. Gassner ${ }^{32}$, J. Gayler ${ }^{10}$, R. Gerhards ${ }^{10}$, S. Ghazaryan ${ }^{34}$, L. Goerlich ${ }^{6}$, N. Gogitidze ${ }^{24}$, M. Goldberg ${ }^{28}$, C. Goodwin ${ }^{3}$, C. Grab ${ }^{36}$, H. Grässler ${ }^{2}$, T. Greenshaw ${ }^{18}$, G. Grindhammer ${ }^{25}$, T. Hadig ${ }^{1}$, D. Haidt ${ }^{10}$, L. Hajduk ${ }^{6}$, V. Haustein ${ }^{33}$, W.J. Haynes ${ }^{5}$, B. Heinemann ${ }^{18}$, G. Heinzelmann ${ }^{11}$, R.C.W. Henderson ${ }^{17}$, S. Hengstmann ${ }^{37}$, H. Henschel ${ }^{35}$, R. Heremans ${ }^{4}$, G. Herrera $^{7,41, k}$, I. Herynek ${ }^{29}$, M. Hilgers ${ }^{36}$, K.H. Hiller ${ }^{35}$, C.D. Hilton ${ }^{21}$, J. Hladký ${ }^{29}$, P. Höting ${ }^{2}$, D. Hoffmann ${ }^{10}$, W. Hoprich ${ }^{12}$, R. Horisberger ${ }^{32}$, S. Hurling ${ }^{10}$, M. Ibbotson ${ }^{21}$, Ç. İşsever ${ }^{7}$, M. Jacquet ${ }^{26}$, M. Jaffre ${ }^{26}$, L. Janauschek ${ }^{25}$, D.M. Jansen ${ }^{12}$, X. Janssen ${ }^{4}$, V. Jemanov ${ }^{11}$, L. Jönsson ${ }^{20}$, D.P. Johnson ${ }^{4}$, M.A.S. Jones ${ }^{18}$, H. Jung ${ }^{20}$, H.K. Kästli ${ }^{36}$, D. Kant ${ }^{19}$, M. Kapichine ${ }^{8}$, M. Karlsson ${ }^{20}$, O. Karschnick ${ }^{11}$, O. Kaufmann ${ }^{13}$, M. Kausch ${ }^{10}$, F. Keil ${ }^{14}$, N. Keller ${ }^{13}$, J. Kennedy ${ }^{18}$, I.R. Kenyon ${ }^{3}$, S. Kermiche ${ }^{22}$, C. Kiesling ${ }^{25}$, M. Klein ${ }^{35}$, C. Kleinwort ${ }^{10}$, G. Knies ${ }^{10}$, B. Koblitz ${ }^{25}$, H. Kolanoski ${ }^{38}$, S.D. Kolya ${ }^{21}$, V. Korbel ${ }^{10}$, P. Kostka ${ }^{35}$, S.K. Kotelnikov ${ }^{24}$, M.W. Krasny ${ }^{28}$, H. Krehbiel ${ }^{10}$, J. Kroseberg ${ }^{37}$, D. Krücker ${ }^{38}$, K. Krüger ${ }^{10}$, A. Küpper ${ }^{33}$, T. Kuhr ${ }^{11}$, T. Kurča ${ }^{35,16}$, R. Kutuev ${ }^{12}$, W. Lachnit ${ }^{10}$, R. Lahmann ${ }^{10}$, D. Lamb ${ }^{3}$, M.P.J. Landon ${ }^{19}$, W. Lange ${ }^{35}$, T. Laštovička ${ }^{30}$, A. Lebedev ${ }^{24}$, B. Leißner ${ }^{1}$, R. Lemrani ${ }^{10}$, V. Lendermann ${ }^{7}$, S. Levonian ${ }^{10}$, M. Lindstroem ${ }^{20}$, G. Lobo ${ }^{26}$, E. Lobodzinska ${ }^{10,6}$, B. Lobodzinski ${ }^{6,10}$, N. Loktionova ${ }^{24}$, V. Lubimov ${ }^{23}$, S. Lüders ${ }^{36}$, D. Lüke ${ }^{7,10}$, L. Lytkin ${ }^{12}$, N. Magnussen ${ }^{33}$, H. Mahlke-Krüger ${ }^{10}$, N. Malden ${ }^{21}$, E. Malinovski ${ }^{24}$, I. Malinovski ${ }^{24}$, R. Maraček ${ }^{25}$, P. Marage ${ }^{4}$, J. Marks ${ }^{13}$, R. Marshall ${ }^{21}$, H.-U. Martyn ${ }^{1}$, J. Martyniak ${ }^{6}$, S.J. Maxfield ${ }^{18}$, A. Mehta ${ }^{18}$, K. Meier ${ }^{14}$, P. Merkel ${ }^{10}$, F. Metlica ${ }^{12}$, A. Meyer ${ }^{10}$, H. Meyer ${ }^{33}$, J. Meyer ${ }^{10}$, P.-O. Meyer ${ }^{2}$, S. Mikocki ${ }^{6}$, D. Milstead ${ }^{18}$, T. Mkrtchyan ${ }^{34}$, R. Mohr ${ }^{25}$, S. Mohrdieck ${ }^{11}$, M.N. Mondragon ${ }^{7}$, F. Moreau ${ }^{27}$, A. Morozov ${ }^{8}$, J.V. Morris ${ }^{5}$, D. Müller ${ }^{37}$, K. Müller ${ }^{13}$, P. Murín ${ }^{16,42}$, V. Nagovizin ${ }^{23}$, B. Naroska ${ }^{11}$, J. Naumann ${ }^{7}$, Th. Naumann ${ }^{35}$, I. Négri ${ }^{22}$, G. Nellen ${ }^{25}$, P.R. Newman ${ }^{3}$, T.C. Nicholls ${ }^{5}$, F. Niebergall ${ }^{11}$, C. Niebuhr ${ }^{10}$, O. Nix ${ }^{14}$, G. Nowak ${ }^{6}$, T. Nunnemann ${ }^{12}$, J.E. Olsson ${ }^{10}$, D. Ozerov ${ }^{23}$, V. Panassik ${ }^{8}$, C. Pascaud ${ }^{26}$, G.D. Patel ${ }^{18}$, E. Perez ${ }^{9}$, J.P. Phillips ${ }^{18}$, D. Pitzl ${ }^{10}$, R. Pöschl ${ }^{7}$, I. Potachnikova ${ }^{12}$, B. Povh ${ }^{12}$, K. Rabbertz ${ }^{1}$, G. Rädel ${ }^{9}$, J. Rauschenberger ${ }^{11}$, P. Reimer ${ }^{29}$, B. Reisert ${ }^{25}$, D. Reyna ${ }^{10}$, S. Riess ${ }^{11}$, E. Rizvi ${ }^{3}$, P. Robmann ${ }^{37}$, R. Roosen ${ }^{4}$, A. Rostovtsev ${ }^{23}$, C. Royon ${ }^{9}$, S. Rusakov ${ }^{24}$, K. Rybicki ${ }^{6}$, D.P.C. Sankey ${ }^{5}$, J. Scheins ${ }^{1}$, F.-P. Schilling ${ }^{13}$, S. Schleif ${ }^{14}$, P. Schleper ${ }^{13}$, D. Schmidt ${ }^{33}$, D. Schmidt ${ }^{10}$, L. Schoeffel ${ }^{9}$, A. Schöning ${ }^{36}$, T. Schörner ${ }^{25}$, V. Schröder ${ }^{10}$, H.-C. Schultz-Coulon ${ }^{10}$, K. Sedlák ${ }^{29}$, F. Sefkow ${ }^{37}$, V. Shekelyan ${ }^{25}$, I. Sheviakov ${ }^{24}$, 
L.N. Shtarkov ${ }^{24}$, G. Siegmon ${ }^{15}$, P. Sievers ${ }^{13}$, Y. Sirois ${ }^{27}$, T. Sloan ${ }^{17}$, P. Smirnov ${ }^{24}$, M. Smith ${ }^{18}$, V. Solochenko ${ }^{23}$, Y. Soloviev ${ }^{24}$, V. Spaskov ${ }^{8}$, A. Specka ${ }^{27}$, H. Spitzer ${ }^{11}$, R. Stamen ${ }^{7}$, J. Steinhart ${ }^{11}$, B. Stella ${ }^{31}$, A. Stellberger ${ }^{14}$, J. Stiewe ${ }^{14}$, U. Straumann ${ }^{37}$, W. Struczinski ${ }^{2}$, M. Swart ${ }^{14}$, M. Taševský ${ }^{29}$, V. Tchernyshov ${ }^{23}, \mathrm{~S}$. Tchetchelnitski ${ }^{23}, \mathrm{G}$. Thompson ${ }^{19}$, P.D. Thompson ${ }^{3}$, N. Tobien ${ }^{10}$, D. Traynor ${ }^{19}$, P. Truöl ${ }^{37}$, G. Tsipolitis ${ }^{36}$, J. Turnau ${ }^{6}$, J.E. Turney ${ }^{19}$, E. Tzamariudaki ${ }^{25}$, S. Udluftt ${ }^{25}$, A. Usik ${ }^{24}$, S. Valkár ${ }^{30}$, A. Valkárováa ${ }^{30}$, C. Vallée ${ }^{22}$, P. Van Mechelen ${ }^{4}$, Y. Vazdik ${ }^{24}, \mathrm{~S}$. von Dombrowski ${ }^{37}, \mathrm{~K}$. Wacker ${ }^{7}, \mathrm{R}$. Wallny ${ }^{13}$, T. Walter ${ }^{37}$, B. Waugh ${ }^{21}$, G. Weber ${ }^{11}$, M. Weber ${ }^{14}$, D. Wegener ${ }^{7}$, A. Wegner ${ }^{11}$, T. Wengler ${ }^{13}$, M. Werner ${ }^{13}$, G. White ${ }^{17}$, S. Wiesand ${ }^{33}$, T. Wilksen ${ }^{10}$, M. Winde ${ }^{35}$, G.-G. Winter ${ }^{10}$, C. Wissing ${ }^{7}$, M. Wobisch ${ }^{2}$, H. Wollatz ${ }^{10}$, E. Wünsch ${ }^{10}$, A.C. Wyatt ${ }^{21}$, J. Žáček ${ }^{30}$, J. Zálešák ${ }^{30}$, Z. Zhang ${ }^{26}$, A. Zhokin ${ }^{23}$, F. Zomer ${ }^{26}$, J. Zsembery ${ }^{9}$ and M. zur Nedden ${ }^{10}$

${ }^{1}$ I. Physikalisches Institut der RWTH, Aachen, Germany ${ }^{a}$

${ }^{2}$ III. Physikalisches Institut der RWTH, Aachen, Germany ${ }^{a}$

${ }^{3}$ School of Physics and Space Research, University of Birmingham, Birmingham, $U K^{b}$

${ }^{4}$ Inter-University Institute for High Energies ULB-VUB, Brussels; Universitaire Instelling Antwerpen, Wilrijk; Belgium ${ }^{c}$

${ }^{5}$ Rutherford Appleton Laboratory, Chilton, Didcot, $U K^{b}$

${ }^{6}$ Institute for Nuclear Physics, Cracow, Poland ${ }^{d}$

${ }^{7}$ Institut für Physik, Universität Dortmund, Dortmund, Germany ${ }^{a}$

${ }^{8}$ Joint Institute for Nuclear Research, Dubna, Russia

${ }^{9}$ DSM/DAPNIA, CEA/Saclay, Gif-sur-Yvette, France

${ }^{10}$ DESY, Hamburg, Germany ${ }^{a}$

${ }^{11}$ II. Institut für Experimentalphysik, Universität Hamburg, Hamburg, Germany ${ }^{a}$

${ }^{12}$ Max-Planck-Institut für Kernphysik, Heidelberg, Germany ${ }^{a}$

${ }^{13}$ Physikalisches Institut, Universität Heidelberg, Heidelberg, Germany ${ }^{a}$

${ }^{14}$ Kirchhoff-Institut für Physik, Universität Heidelberg, Heidelberg, Germany ${ }^{a}$

${ }^{15}$ Institut für experimentelle und angewandte Physik, Universität Kiel, Kiel, Germany ${ }^{a}$

${ }^{16}$ Institute of Experimental Physics, Slovak Academy of Sciences, Košice, Slovak Republic e,f

${ }^{17}$ School of Physics and Chemistry, University of Lancaster, Lancaster, UK ${ }^{b}$

${ }^{18}$ Department of Physics, University of Liverpool, Liverpool, $U K^{b}$

${ }^{19}$ Queen Mary and Westfield College, London, $U K^{b}$

${ }^{20}$ Physics Department, University of Lund, Lund, Sweden ${ }^{g}$

${ }^{21}$ Department of Physics and Astronomy, University of Manchester, Manchester, $U K^{b}$

${ }^{22}$ CPPM, CNRS/IN2P3 - Univ Mediterranee, Marseille - France

${ }^{23}$ Institute for Theoretical and Experimental Physics, Moscow, Russia

${ }^{24}$ Lebedev Physical Institute, Moscow, Russia ${ }^{e, h}$

${ }^{25}$ Max-Planck-Institut für Physik, München, Germany ${ }^{a}$

${ }^{26}$ LAL, Université de Paris-Sud, IN2P3-CNRS, Orsay, France

${ }^{27}$ LPNHE, École Polytechnique, IN2P3-CNRS, Palaiseau, France

${ }^{28}$ LPNHE, Universités Paris VI and VII, IN2P3-CNRS, Paris, France

${ }^{29}$ Institute of Physics, Academy of Sciences of the Czech Republic, Praha, Czech Republic ${ }^{e, i}$

${ }^{30}$ Faculty of Mathematics and Physics, Charles University, Praha, Czech Republic ${ }^{e, i}$

${ }^{31}$ INFN Roma 1 and Dipartimento di Fisica, Università Roma 3, Roma, Italy

32 Paul Scherrer Institut, Villigen, Switzerland 
${ }^{33}$ Fachbereich Physik, Bergische Universität Gesamthochschule Wuppertal, Wuppertal, Germany $^{a}$

${ }^{34}$ Yerevan Physics Institute, Yerevan, Armenia

${ }^{35}$ DESY, Zeuthen, Germany ${ }^{a}$

${ }^{36}$ Institut für Teilchenphysik, ETH, Zürich, Switzerland ${ }^{j}$

${ }^{37}$ Physik-Institut der Universität Zürich, Zürich, Switzerland ${ }^{j}$

38 Present address: Institut für Physik, Humboldt-Universität, Berlin, Germany

${ }^{39}$ Also at Rechenzentrum, Bergische Universität Gesamthochschule Wuppertal, Wuppertal, Germany

${ }^{40}$ Also at Institut für Experimentelle Kernphysik, Universität Karlsruhe, Karlsruhe, Germany

${ }^{41}$ Also at Dept. Fis. Ap. CINVESTAV, Mérida, Yucatán, México ${ }^{k}$

${ }^{42}$ Also at University of P.J. Šafárik, Košice, Slovak Republic

${ }^{43}$ Also at CERN, Geneva, Switzerland

$\dagger$ Deceased

${ }^{a}$ Supported by the Bundesministerium für Bildung, Wissenschaft, Forschung und Technologie, FRG, under contract numbers 7AC17P, 7AC47P, 7DO55P, 7HH17I, 7HH27P, 7HD17P, 7HD27P, 7KI17I, 6MP17I and 7WT87P

${ }^{b}$ Supported by the UK Particle Physics and Astronomy Research Council, and formerly by the UK Science and Engineering Research Council

${ }^{c}$ Supported by FNRS-FWO, IISN-IIKW

${ }^{d}$ Partially Supported by the Polish State Committee for Scientific Research, grant No. 2P0310318 and SPUB/DESY/P-03/DZ 1/99

${ }^{e}$ Supported by the Deutsche Forschungsgemeinschaft

${ }^{f}$ Supported by VEGA SR grant no. 2/5167/98

${ }^{g}$ Supported by the Swedish Natural Science Research Council

${ }^{h}$ Supported by Russian Foundation for Basic Research grant no. 96-02-00019

${ }^{i}$ Supported by GA AVČR grant number no. A1010821

${ }^{j}$ Supported by the Swiss National Science Foundation

${ }^{k}$ Supported by CONACyT 


\section{Introduction}

The HERA collider enables the study of deep inelastic neutral current scattering $e p \rightarrow e X$ at very high squared momentum transfers $Q^{2}$, thus probing the structure of $e q$ interactions at very short distances. At large scales new phenomena not directly detectable may become observable as deviations from the Standard Model predictions. A convenient tool to assess the experimental sensitivity beyond the maximal available center of mass energy and to parameterise indirect signatures of new physics is the concept of four-fermion contact interactions. Possible sources of such contact terms are either a substructure of the fermions involved or the exchange of a new heavy particle. In the first case a compositeness scale can be related to the size of the composite object, while in the second case the scale parameter is related to the mass and coupling of the exchanged boson.

The principle idea of this contact interaction analysis at HERA is to fix the Standard Model and its parameters, in particular the parton distributions, using experimental data at low $Q^{2}$, where the theory is well established, and to extrapolate the prediction towards high momentum transfers, where deviations due to new physics are expected to be most prominent. In the present paper the differential cross section $\mathrm{d} \sigma / \mathrm{d} Q^{2}$ is analysed over a $Q^{2}$ range of $200-30,000 \mathrm{GeV}^{2}$ and possible deviations from the Standard Model prediction are searched for in the framework of $(\bar{e} e)(\bar{q} q)$ contact interactions. The data are interpreted within conventional scenarios such as model independent compositeness scales of various chiral structures, a classical quark form factor approach and the exchange of heavy leptoquarks. Another investigation concerns the search for low scale quantum gravity effects, which may be observable at HERA via the exchange of gravitons coupling to Standard Model particles and propagating into extra spatial dimensions.

\section{Data Analysis}

The contact interaction analysis is based on the recent $e^{+} p$ neutral current cross section measurements by the $\mathrm{H} 1$ experiment discussed in detail in ref. [1]. The data have been collected at a center of mass energy of $\sqrt{s}=300 \mathrm{GeV}$ and correspond to an integrated luminosity of $\mathcal{L}=35.6 \mathrm{pb}^{-1}$, representing a tenfold increase over a previous analysis [2]. The cross section $\mathrm{d} \sigma / \mathrm{d} Q^{2}$ is determined from a purely inclusive measurement of the final state positron with energy $E_{e}^{\prime}$ and polar angle $\theta_{e}$ (defined with respect to the proton direction). The squared momentum transfer is calculated via $Q^{2}=4 E_{e} E_{e}^{\prime} \cos ^{2}\left(\theta_{e} / 2\right)$, where $E_{e}$ is the lepton beam energy. The data are corrected for detector effects and QED radiation and represent the cross section within the kinematic phase space of momentum transfer $Q^{2} \geq 200 \mathrm{GeV}^{2}$ and inelasticity

$y=1-E_{e}^{\prime} / E_{e} \sin ^{2}\left(\theta_{e} / 2\right)<0.9$. The dominant experimental systematics are the uncertainties of the reconstructed positron energy scale, varying between $0.7 \%$ and $3 \%$ depending on the detector region, and of the scattering angle, known to $1-3 \mathrm{mrad}$. An overall normalisation uncertainty of $1.5 \%$ is due to the luminosity determination. The differential cross section is shown in figure 1.

The double differential cross section is given in the Standard Model by

$$
\frac{\mathrm{d}^{2} \sigma\left(e^{+} p \rightarrow e^{+} X\right)}{\mathrm{d} x \mathrm{~d} Q^{2}}=\frac{2 \pi \alpha^{2}}{x Q^{4}}\left\{Y_{+} F_{2}\left(x, Q^{2}\right)-Y_{-} x F_{3}\left(x, Q^{2}\right)-y^{2} F_{L}\left(x, Q^{2}\right)\right\},
$$


where $x=Q^{2} / y s$ is the Bjorken scaling variable and $Y_{ \pm}=1 \pm(1-y)^{2}$. The generalised proton structure functions $F_{2}\left(x, Q^{2}\right), F_{3}\left(x, Q^{2}\right)$ and $F_{L}\left(x, Q^{2}\right)$ are related to the parton densities and the quark- $\gamma$ and quark- $Z$ couplings. The cross section calculations are done in the DIS scheme in next-to-leading-order using as standard the CTEQ5D parton parameterisation [3]. Integrating eq. (11) over $x$ gives the $Q^{2}$ spectrum which describes the data very well over six orders of magnitude, see figure 1.

In order to derive quantitative tests of the Standard Model and to search for new physics hypotheses, a $\chi^{2}$ analysis of the data is performed taking the dominant error sources and uncertainties into account. The $\chi^{2}$ function is defined as

$$
\chi^{2}=\sum_{i}\left(\frac{\hat{\sigma}_{i}^{e x p} f_{n}-\hat{\sigma}_{i}^{t h}\left(1-\sum_{k} \Delta_{i k}\left(\varepsilon_{k}\right)\right)}{\Delta \hat{\sigma}_{i}^{e x p} f_{n}}\right)^{2}+\left(\frac{f_{n}-1}{\Delta f_{n}}\right)^{2}+\sum_{k} \varepsilon_{k}^{2} .
$$

Here $\hat{\sigma}_{i}$ denotes the experimental or theoretical cross section in the $Q^{2}$ bin $i$ and $f_{n}$ is the overall normalisation parameter with an uncertainty $\Delta f_{n}=0.015$. The experimental error $\Delta \hat{\sigma}_{i}^{e x p}$ includes statistical and uncorrelated systematic errors added in quadrature. The functions $\Delta_{i k}\left(\varepsilon_{k}\right)$ describe for the $i^{\text {th }}$ bin effects due to correlated systematic errors associated to different sources $k$. They depend quadratically on the fit parameters $\varepsilon_{k}$, which may be interpreted as pulls, i.e. shifts caused by systematics normalised to their error estimates. There are three sources of correlated systematic errors taken into account: the experimental uncertainties of the positron energy scale and the scattering angle and the uncertainty of the strong coupling entering in the Standard Model prediction (see below).

Concerning cross section calculations the major uncertainty comes from the parton distributions, which are generally provided without error estimates. Different parametrisations in the DIS scheme, MRST 99 [4] and GRV 94 [5] in addition to CTEQ5D, are used to estimate the uncertainties due to various models and assumptions. They do not differ in the shape of the $Q^{2}$ spectrum significantly, but rather in the absolute cross section prediction by up to $2.8 \%$, e.g. comparing CTEQ5D with MRST 99. Several other MRST sets are used for cross checks, like those with different admixtures of quarks and gluons at high $x$, or different treatments of strange and charm quarks. All these MRST variants essentially change the overall normalisation of the cross section prediction by less than $1 \%$, being well below the measurement errors, and introduce no relevant additional $Q^{2}$ dependence. The largest uncertainty comes from the strong coupling constant. Using parton distributions evaluated for couplings differing from the central value of $\alpha_{s}\left(M_{Z}\right)=0.118$ by \pm 0.005 cause variations of the cross section by $\pm 1 \%$ at low $Q^{2}$ and up to $\mp 4 \%$ at high $Q^{2}$. These shifts are parameterised and taken into account as correlated systematic error in the $\chi^{2}$ fit of eq. (2). It should be noted that the applied parton density functions have not been constrained by high $Q^{2}$ data from the HERA experiments. A comparison with a recent QCD analysis in the $\overline{\mathrm{MS}}$ scheme [6], which attempts to provide parton distributions including errors, confirms that the above choice of various parton density functions is a reasonable representation of systematic uncertainties.

A fit of the cross section $\mathrm{d} \sigma / \mathrm{d} Q^{2}$ to the Standard Model expectation using CTEQ5D parton densities yields $\chi^{2} /$ dof $=12.3 / 16$ with a normalisation parameter $f_{n}=1.004$. Limits of a model parameter are derived by varying the parameter until the $\chi^{2}$ value changes by a certain amount with respect to the Standard Model fit, e.g. $\chi^{2}-\chi_{S M}^{2}=3.84$ for $95 \%$ confidence level (CL). Systematics due to different parton distributions are taken into account by always quoting the most conservative result of the various fits, i.e. the smallest value in case of a lower limit. 


\section{Contact Interaction Phenomenology}

New currents or heavy bosons may produce indirect effects through the exchange of a virtual particle interfering with the $\gamma$ and $Z$ fields of the Standard Model. For particle masses and scales well above the available energy, $\Lambda \gg \sqrt{s}$, such indirect signatures may be investigated by searching for a four-fermion pointlike $(\bar{e} e)(\bar{q} q)$ contact interaction. The most general chiral invariant Lagrangian for neutral current vector-like contact interactions can be written in the form [7, 8]

$$
\begin{array}{r}
\mathcal{L}_{V}=\sum_{q=u, d}\left\{\eta_{L L}^{q}\left(\bar{e}_{L} \gamma_{\mu} e_{L}\right)\left(\bar{q}_{L} \gamma^{\mu} q_{L}\right)+\eta_{L R}^{q}\left(\bar{e}_{L} \gamma_{\mu} e_{L}\right)\left(\bar{q}_{R} \gamma^{\mu} q_{R}\right)\right. \\
\left.\quad+\eta_{R L}^{q}\left(\bar{e}_{R} \gamma_{\mu} e_{R}\right)\left(\bar{q}_{L} \gamma^{\mu} q_{L}\right)+\eta_{R R}^{q}\left(\bar{e}_{R} \gamma_{\mu} e_{R}\right)\left(\bar{q}_{R} \gamma^{\mu} q_{R}\right)\right\}
\end{array}
$$

where the indices $L$ and $R$ denote the left-handed and right-handed fermion helicities and the sum extends over up-type and down-type quarks and antiquarks $q$. In deep inelastic scattering at high $Q^{2}$ the contributions from the first generation $u$ and $d$ quarks completely dominate and contact terms arising from sea quarks $s, c$ and $b$ are strongly suppressed. Thus, there are eight independent effective coupling coefficients, four for each quark flavour

$$
\eta_{a b}^{q} \equiv \epsilon \frac{g^{2}}{\Lambda_{a b}^{q 2}},
$$

where $a$ and $b$ indicate the $L, R$ helicities, $g$ is the overall coupling strength, $\Lambda_{a b}^{q}$ is a scale parameter and $\epsilon$ is a prefactor, often set to $\epsilon= \pm 1$, which determines the interference sign with the Standard Model currents. The ansatz eq. (3) can be easily applied to any new phenomenon, e.g. (eq) compositeness, leptoquarks or new gauge bosons, by an appropriate choice of the coefficients $\eta_{a b}$. Scalar and tensor interactions of dimension 6 operators involving helicity flip couplings are strongly suppressed at HERA [8] and therefore not considered.

It has been recently suggested that gravitational effects may become strong at subatomic distances and thus measurable in collider experiments [9]. In such a scenario, which may be realised in string theory, gravity is characterised by a scale $M_{S} \sim \mathcal{O}(\mathrm{TeV})$ in $4+n$ dimensions. The extra spatial dimensions $n$ are restricted to a volume associated with the size $R$ and the scales in $4+n$ and the ordinary 4 dimensions are related by

$$
M_{P}^{2} \sim R^{n} M_{S}^{2+n},
$$

where $M_{P} \sim 10^{19} \mathrm{GeV}$ is the Planck mass. An exciting consequence would be a modification of Newton's law at distances $r<R$, where the gravitational force would rise rapidly as $F \propto$ $1 / r^{2+n}$ and become strong at the scale $M_{S}$. Experimentally, gravity is essentially not tested in the sub-millimeter range [10] and scenarios with $n>2$ extra dimensions at large distances $R \lesssim 100 \mu \mathrm{m}$ are conceivable.

In the phenomenology of [11] the Standard Model particles are confined to 4 dimensions while only the graviton propagates as massless spin 2 particle into the $n$ extra dimensions. When projected onto the normal 4 dimensional space the graviton appears as a spectrum of Kaluza-

Klein excitations with masses $m^{(j)}=j / R$, including the zero-mass state. The graviton fields $G_{\mu \nu}^{(j)}$ couple to the Standard Model particles via the energy-momentum tensor $T_{\mu \nu}$

$$
\mathcal{L}_{G}=-\frac{\sqrt{8 \pi}}{M_{P}} G_{\mu \nu}^{(j)} T^{\mu \nu} .
$$


Summation over the whole tower of Kaluza-Klein states $j$ with masses up to the scale $M_{S}$ compensates the huge $1 / M_{P}$ suppression and results in an effective contact interaction coupling

$$
\eta_{G}=\frac{\lambda}{M_{S}^{4}},
$$

where $\lambda$ is the coupling strength of order unity. Note that the scale dependence of gravitational effects is very different from that of conventional contact interactions, eq. (4). In deep inelastic scattering graviton exchange may contribute to the electron-quark subprocess, but the new interaction also induces electron-gluon scattering which is not present in the Standard Model.

It is worth recalling that contact interactions as an effective theory can only be formulated in lowest order. They contribute [8] to the structure functions $F_{2}\left(x, Q^{2}\right)$ and $x F_{3}\left(x, Q^{2}\right)$, but are absent in $F_{L}\left(x, Q^{2}\right)$. On the other hand a cross section calculation in next-to-leading-order QCD appears to be more reliable. This conceptual limitation is less important in the DIS renormalisation scheme, where the expression for the dominant structure function $F_{2}$ is identical and $x F_{3}$ receives only minor corrections in second order.

Contact interaction phenomena are best observed as a modification of the expected $Q^{2}$ dependence and all information is essentially contained in the differential cross section $\mathrm{d} \sigma / \mathrm{d} Q^{2}$, analysed in the present paper. Calculations using the Standard Model prediction, eq. (11), show that for the scenarios under study only those models involving both $u$ and $d$ quarks with pure $L L$ or $R R$ couplings and negative interference are slightly more sensitive to an analysis in two variables $Q^{2}$ and $x$. With the present luminosity the gain in setting limits on the respective scales would be $\sim 20 \%$ for the negatively interfering $L L$ and $R R$ composite models and $\sim 10 \%$ for the leptoquark $S_{1}^{L}$. For all other scenarios the differences are marginal.

\section{Compositeness Scales}

In the Standard Model the fundamental particles - leptons, quarks and gauge bosons - are assumed to be pointlike. A possible fermion compositeness or substructure can be expressed through the $\eta$ coefficients of eq. (4) which depend only on the ratio of the coupling constant over the scale. In the present analysis the interference sign is set to $\epsilon= \pm 1$ for the chiral structures under study, the coupling strength $g$ is by convention chosen as $g^{2}=4 \pi$ and the compositeness scale $\Lambda$ is assumed to be the same for all up-type and down-type quarks. Among the many possible combinations the following models are investigated: (i) the pure chiral couplings $L L$, $L R, R L$ and $R R$, (ii) the vectorial couplings $V V, A A$ and $V A$, (iii) the mixtures $L L+R R$ and $L R+R L$.

It is appropriate to analyse the differential cross section in terms of the coupling coefficients $\eta=\epsilon 4 \pi / \Lambda^{2}$. Figure 2 shows the values of $\chi^{2}$ as a function of $\epsilon / \Lambda^{2}$ from fits to the models under study. In general one observes that the distributions become narrower, i.e. the sensitivity increases, the more chiral structures are involved. The pure chiral couplings prefer negative values of $\eta$. This is a consequence of the trend of the data $\left(\mathrm{d} \sigma / \mathrm{d} Q^{2}\right) /\left(\mathrm{d} \sigma^{S M} / \mathrm{d} Q^{2}\right)$ to be slightly low around $Q^{2} \simeq 4,000-12,000 \mathrm{GeV}^{2}$ and being followed by an upward fluctuation

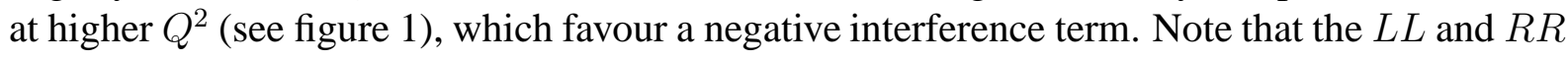


models and the $L R$ and $R L$ models are almost indistinguishable in deep inelastic unpolarised $e^{+} p$ scattering. Within each couple the exchanged quantum numbers are the same and therefore the combinations $L L+R R$ and $L R+R L$ are investigated as well. The data are more sensitive to the $V V, A A$ and $V A$ models, where all chiral structures contribute. The most restrictive range of $\epsilon / \Lambda^{2}$ is obtained for the $V V$ model, where all contact terms enter with the same sign. Figure 2 also shows that different parton distributions have little influence on the results.

\begin{tabular}{lccc}
\hline coupling & $\epsilon / \Lambda^{2}\left[\mathrm{TeV}^{-2}\right]$ & $\Lambda^{+}[\mathrm{TeV}]$ & $\Lambda^{-}[\mathrm{TeV}]$ \\
\hline$L L$ & $-0.249_{-0.207-0.000}^{+0.235+0.069}$ & 2.4 & 1.3 \\
$L R$ & $-0.247_{-0.064-0.012}^{+0.121+0.000}$ & 3.4 & 1.6 \\
$R L$ & $-0.226_{-0.067-0.006}^{+0.130+0.000}$ & 3.4 & 1.6 \\
$R R$ & $-0.251_{-0.209-0.000}^{+0.228+0.068}$ & 2.5 & 1.3 \\
$V V$ & $-0.028_{-0.033-0.004}^{+0.027+0.008}$ & 5.5 & 2.8 \\
$A A$ & $0.131_{-0.109-0.000}^{+0.044+0.013}$ & 2.1 & 3.9 \\
$V A$ & $-0.007_{-0.071-0.005}^{+0.084+0.004}$ & 2.8 & 2.8 \\
$L L+R R$ & $-0.119_{-0.236-0.013}^{+0.106+0.041}$ & 3.3 & 1.4 \\
$L R+R L$ & $-0.046_{-0.154-0.010}^{+0.046+0.017}$ & 4.6 & 1.8 \\
\hline
\end{tabular}

Table 1: Results of $\chi^{2}$ fits of the parameter $\epsilon / \Lambda^{2}$ for various chiral structures using CTEQ5D parton distributions with 68\% CL intervals (first error) and variations due to MRST 99 and GRV 94 parton parametrisations (second error). The compositeness scale parameters $\Lambda^{ \pm}$ (95\% CL lower limits) are derived as weakest bounds, i.e. smallest values from the analyses applying different parton densities.

The results of the $\chi^{2}$ fits are shown in figure 3 and compiled in table 1. Within two standard deviations the couplings $\epsilon / \Lambda^{2}$ are compatible with the Standard Model for all parton density functions used.

Limits on the compositeness scale parameters $\Lambda^{+}$and $\Lambda^{-}$, corresponding to positive and negative interference, are quoted in table 1 and also presented in figure 3 . They vary between 1.3 TeV and 5.5 TeV depending on the chiral structure of the model and are in most cases asymmetric with respect to the interference sign. In general the values of $\Lambda^{+}$are more restrictive due to the downward trend of the data at intermediate $Q^{2}$ prefering models with negative interference. As an illustration of the sensitivity of the data to compositeness scales figure 4 shows the 95\% CL contributions of $\Lambda^{ \pm}$for the $V V$ model using CTEQ5D parton densities.

The results of direct searches for $(e q)$ compositeness are compatible with those of other experiments at HERA, LEP and TEVATRON. To date the most stringent limits come from $e^{+} e^{-}$ experiments [12] with typical cut-off values of $3-10 \mathrm{TeV}$ assuming, however, in general the same scale parameter $\Lambda$ for all five active quarks. The ZEUS collaboration [13] investigates only models in which at least 2 couplings contribute and derives limits between $1.7 \mathrm{TeV}$ and $5 \mathrm{TeV}$ relying solely on the shape of measured distributions. The $p \bar{p}$ experiments [14] measure DrellYan production and quote limits on $\Lambda$ between $2.5 \mathrm{TeV}$ and $6 \mathrm{TeV}$, where the normalisation is 
based on the number of observed $Z$ bosons. Model dependent indirect limits of order $10 \mathrm{TeV}$ for the pure chiral couplings involving $u$ and $d$ quarks can be set by atomic parity violation experiments [15].

\section{Form Factors}

An alternative method to study possible fermion substructures is to assign a finite size of radius $R$ to the electroweak charges of leptons and/or quarks while treating the gauge bosons $\gamma$ and $Z$ still as pointlike particles [16]. A convenient parametrisation is to introduce 'classical' form factors $f\left(Q^{2}\right)$ at the gauge boson-fermion vertices, which are expected to diminish the Standard Model cross section at high momentum transfer

$$
\begin{aligned}
f\left(Q^{2}\right) & =1-\frac{1}{6}\left\langle r^{2}\right\rangle Q^{2} \\
\frac{d \sigma}{d Q^{2}} & =\frac{d \sigma^{S M}}{d Q^{2}} f_{e}^{2}\left(Q^{2}\right) f_{q}^{2}\left(Q^{2}\right) .
\end{aligned}
$$

The root of the mean-square radius of the electroweak charge distribution, $R=\sqrt{\left\langle r^{2}\right\rangle}$, is taken as a measure of the particle size. The data are analysed in terms of a single form factor $f_{q}$, i.e. only the quarks are allowed to be extended objects while the positron has no structure by setting $f_{e} \equiv 1$. This assumption is justified, since the pointlike nature of the electron/positron is already established down to extremely low distances in $e^{+} e^{-}$and $(g-2)_{e}$ experiments [17]. The analysis yields an upper limit at $95 \% \mathrm{CL}$ of the light quark radius of

$$
R_{q}<1.7 \cdot 10^{-16} \mathrm{~cm}
$$

The result is compatible with those from other measurements. In an analysis of DrellYan production of $e^{+} e^{-}$and $\mu^{+} \mu^{-}$pairs in $p \bar{p}$ scattering the CDF collaboration [14] finds a quark size of $R_{q}<1 \cdot 10^{-16} \mathrm{~cm}$ assuming pointlike leptons. A complementary analysis of the contributions of anomalous magnetic dipole moments to the $Z q \bar{q}$ vertex using hadronic $Z$ decays gives $R_{q}<1.2 \cdot 10^{-16} \mathrm{~cm}$ for the light $u$ and $d$ quarks [16].

\section{Leptoquarks}

Leptoquarks are colour triplet bosons of spin 0 or 1, carrying lepton $(L)$ and baryon $(B)$ number and fractional electric charge. They couple to lepton-quark pairs and appear in extensions of the Standard Model which try to establish a connection between leptons and quarks. Leptons and quarks may be arranged in common multiplets, like in Grand Unified Theories or superstring motivated $E_{6}$ models, or they may have a common substructure as in composite models. A fermion number $F=L+3 B$ is defined, which takes the values $F=2$ for leptoquarks coupling to $e^{-} q$ and $F=0$ for leptoquarks coupling to $e^{-} \bar{q}$. For positrons the fermion number $F$ changes by two. The leptoquark mass $M_{L Q}$ and its coupling $\lambda$ are related to the contact interaction 
coefficients of eq. (4) via $g / \Lambda=\lambda / M_{L Q}$. The notation and the coupling coefficients $\eta_{a b}$ for leptoquarks 1 are given in table 2. The only unknown parameter is the ratio $M_{L Q} / \lambda$. Note that the vector leptoquarks have coupling coefficients twice as large in magnitude compared to scalar leptoquarks.

\begin{tabular}{ccccc}
\hline leptoquark & $\begin{array}{c}\text { coupling to } u \text { quark } \\
{\left[\mathrm{GeV}^{-2}\right]}\end{array}$ & $\begin{array}{c}\text { coupling to } d \text { quark } \\
{\left[\mathrm{GeV}^{-2}\right]}\end{array}$ & $\begin{array}{c}F \\
{[\mathrm{GeV}]}\end{array}$ \\
\hline$S_{0}^{L}$ & $\eta_{L L}^{u}=+\frac{1}{2}\left(\lambda / M_{L Q}\right)^{2}$ & 2 & 620 \\
$S_{0}^{R}$ & $\eta_{R R}^{u}=+\frac{1}{2}\left(\lambda / M_{L Q}\right)^{2}$ & & 570 \\
$\tilde{S}_{0}^{R}$ & & $\eta_{R R}^{d}=+\frac{1}{2}\left(\lambda / M_{L Q}\right)^{2}$ & 2 & 220 \\
$S_{1 / 2}^{L}$ & $\eta_{L R}^{u}=-\frac{1}{2}\left(\lambda / M_{L Q}\right)^{2}$ & & 0 & 340 \\
$S_{1 / 2}^{R}$ & $\eta_{R L}^{u}=-\frac{1}{2}\left(\lambda / M_{L Q}\right)^{2}$ & $\eta_{R L}^{d}=-\frac{1}{2}\left(\lambda / M_{L Q}\right)^{2}$ & 0 & 320 \\
$\tilde{S}_{1 / 2}^{L}$ & & $\eta_{L R}^{d}=-\frac{1}{2}\left(\lambda / M_{L Q}\right)^{2}$ & 0 & 450 \\
$S_{1}^{L}$ & $\eta_{L L}^{u}=+\frac{1}{2}\left(\lambda / M_{L Q}\right)^{2}$ & $\eta_{L L}^{d}=+1\left(\lambda / M_{L Q}\right)^{2}$ & 2 & 420 \\
\hline$V_{0}^{L}$ & & $\eta_{L L}^{d}=-1\left(\lambda / M_{L Q}\right)^{2}$ & 0 & 670 \\
$V_{0}^{R}$ & & $\eta_{R R}^{d}=-1\left(\lambda / M_{L Q}\right)^{2}$ & 0 & 550 \\
$\tilde{V}_{0}^{R}$ & $\eta_{R R}^{u}=-1\left(\lambda / M_{L Q}\right)^{2}$ & & 0 & 410 \\
$V_{1 / 2}^{L}$ & & $\eta_{L R}^{d}=+1\left(\lambda / M_{L Q}\right)^{2}$ & 2 & 380 \\
$V_{1 / 2}^{R}$ & $\eta_{R L}^{u}=+1\left(\lambda / M_{L Q}\right)^{2}$ & $\eta_{R L}^{d}=+1\left(\lambda / M_{L Q}\right)^{2}$ & 2 & 960 \\
$\tilde{V}_{1 / 2}^{L}$ & $\eta_{L R}^{u}=+1\left(\lambda / M_{L Q}\right)^{2}$ & & 2 & 1060 \\
$V_{1}^{L}$ & $\eta_{L L}^{u}=-2\left(\lambda / M_{L Q}\right)^{2}$ & $\eta_{L L}^{d}=-1\left(\lambda / M_{L Q}\right)^{2}$ & 0 & 450 \\
\hline
\end{tabular}

Table 2: Coupling coefficients $\eta_{a b}^{q}$, fermion number $F$ and 95\% CL lower limits on $M_{L Q} / \lambda$ for scalar (S) and vector (V) leptoquarks, taking the most conservative values from a variation of different parton distributions. The notation indicates the lepton chirality $L, R$ and weak isospin $I=0,1 / 2,1 . \tilde{S}$ and $\tilde{V}$ differ by two units of hypercharge from $S$ and $V$. By convention the quantum numbers and helicities are given for $e^{-} q$ and $e^{-} \bar{q}$ states. Limits on the coupling $\lambda$ are only meaningful for leptoquark masses $M_{L Q}>\sqrt{s}$.

The differential cross section analysis gives no evidence for a virtual leptoquark signal. The resulting lower limits on $M_{L Q} / \lambda$ are summarised in table 2 including the full error propagation and a variation of parton densities. In general leptoquarks with positive interference provide stronger limits compared to those with negative interference. This observation is consistent with the results found for compositeness models. The vector leptoquarks which couple to $u$ quarks provide the most restrictive limits of $M_{L Q} / \lambda \sim \mathcal{O}(1 \mathrm{TeV})$. It should be emphasised that upper bounds on the coupling strength $\lambda$ can only be set for leptoquark masses exceeding the accessible center of mass energy of HERA. Masses far above $300 \mathrm{GeV}$ are excluded for almost all types of leptoquarks with a coupling of $\lambda \gtrsim 1$.

\footnotetext{
${ }^{1}$ The coupling coefficients are taken from ref. [8] with the signs corrected (i.e. reversed) for $F=2$ scalar and $F=0$ vector leptoquarks according to ref. [18].
} 
These measurements are a considerable improvement over the previous analysis [2]. But it should be noted that changes [18] in the signs of couplings $\eta_{a b}^{q}$ reduce the sensitivity for $F=2$ vector leptoquarks and lead $e . g$. to weaker limits for $\tilde{V}_{0}^{R}$ and $V_{1}^{L}$ despite the increased luminosity. As an example of the sensitivity of the data to virtual leptoquark exchange figure 5 shows the contributions given by the lower limits on $M_{L Q} / \lambda$ for the scalar leptoquark $S_{1 / 2}^{R}$ and the vector leptoquark $V_{1 / 2}^{R}$. Both leptoquarks have $R L$ couplings to up and down quarks, which differ in magnitude and interference sign.

The present contact interaction results complement the direct leptoquark searches of the H1 collaboration [19], which have recently been extended beyond the kinematic reach of HERA up to masses of $M_{L Q} \simeq 400 \mathrm{GeV}$. The coupling limits derived in both analyses are compatible with each other in the mass region where they overlap. Virtual leptoquark exchange has also been studied in $e^{+} e^{-}$annihilation experiments at LEP [12]. Typical limits on $M_{L Q} / \lambda$ are in the range $0.3-1.8 \mathrm{TeV}$, but the sensitivity to particular leptoquark types is different from deep inelastic $e^{+} p$ scattering. In most cases the LEP results provide more stringent bounds; the limits for $S_{0}^{R}, \tilde{S}_{1 / 2}^{L}$ and $V_{0}^{R}$ are comparable and those of $V_{1 / 2}^{R}$ and $\tilde{V}_{1 / 2}^{L}$ are superior at HERA.

\section{Large Extra Dimensions}

The contributions of virtual graviton exchange to deep inelastic scattering have been derived from the cross sections given in ref. [11] for $e^{+} e^{-}$collisions by applying crossing relations. The basic processes of elastic $e^{+} q \rightarrow e^{+} q$ and $e^{+} g \rightarrow e^{+} g$ scattering can be written as f $^{2}$

$$
\begin{aligned}
\frac{\mathrm{d} \sigma\left(e^{+} q \rightarrow e^{+} q\right)}{\mathrm{d} t} & =\frac{\mathrm{d} \sigma^{S M}}{\mathrm{~d} t}+\frac{\mathrm{d} \sigma^{G}}{\mathrm{~d} t}+\frac{\mathrm{d} \sigma^{\gamma G}}{\mathrm{~d} t}+\frac{\mathrm{d} \sigma^{Z G}}{\mathrm{~d} t}, \\
\frac{\mathrm{d} \sigma^{G}}{\mathrm{~d} t} & =\frac{\pi \lambda^{2}}{32 M_{S}^{8}} \frac{1}{s^{2}}\left\{32 u^{4}+64 u^{3} t+42 u^{2} t^{2}+10 u t^{3}+t^{4}\right\} \\
\frac{\mathrm{d} \sigma^{\gamma G}}{\mathrm{~d} t} & =-\frac{\pi \lambda}{2 M_{S}^{4}} \frac{\alpha e_{q}}{s^{2}} \frac{(2 u+t)^{3}}{t}, \\
\frac{\mathrm{d} \sigma^{Z G}}{\mathrm{~d} t} & =\frac{\pi \lambda}{2 M_{S}^{4}} \frac{\alpha}{s^{2} \sin ^{2} 2 \theta_{W}}\left\{v_{e} v_{q} \frac{(2 u+t)^{3}}{t-m_{Z}^{2}}-a_{e} a_{q} \frac{t\left(6 u^{2}+6 u t+t^{2}\right)}{t-m_{Z}^{2}}\right\} \\
\frac{\mathrm{d} \sigma\left(e^{+} g \rightarrow e^{+} g\right)}{\mathrm{d} t} & =\frac{\pi \lambda^{2}}{8 M_{S}^{8}} \frac{u}{s^{2}}\left\{2 u^{3}+4 u^{2} t+3 u t^{2}+t^{3}\right\}
\end{aligned}
$$

in an obvious notation of Standard Model (SM), pure graviton (G), $\gamma G$ and $Z G$ interference contributions. Here $s, t=-Q^{2}$ and $u$ are the Mandelstam variables, $e_{q}$ is the quark charge and $v_{f}$ and $a_{f}$ are the vector and axial vector couplings of the fermions to the $Z$. The corresponding cross sections for $e^{+} \bar{q}$ scattering are obtained by replacing $e_{q} \rightarrow-e_{q}$ and $v_{q} \rightarrow-v_{q}$ in the

\footnotetext{
${ }^{2}$ The following formulae of ref. [11] are used: eq. (79) for the $e q$ contributions, eq. (77) for the $e g$ contribution replacing the photons by gluons, and eqs. (A.5), (A.7) - (A.9) to expand the functions $G_{i}(s / t)$. The present $e q$ results are in agreement with [20], but the $e g$ part differs by a factor of $1 / 4$. The cross section formulae of refs. [21] and [22] cannot be confirmed and the results of both publications are inconsistent with each other.
} 
expressions above. In order to get the inclusive $e^{+} p$ cross section the subprocesses have to be integrated over the $x$ dependent parton distributions, $q(x), \bar{q}(x)$ and $g(x)$, and to be summed up

$$
\frac{\mathrm{d} \sigma\left(e^{+} p \rightarrow e^{+} X\right)}{\mathrm{d} Q^{2}}=\int \mathrm{d} x\left\{q(x) \frac{\mathrm{d} \sigma\left(e^{+} q\right)}{\mathrm{d} t}+\bar{q}(x) \frac{\mathrm{d} \sigma\left(e^{+} \bar{q}\right)}{\mathrm{d} t}+g(x) \frac{\mathrm{d} \sigma\left(e^{+} g\right)}{\mathrm{d} t}\right\}
$$

Note, that expected gravitational effects arising from the gluon contribution are for the highest $Q^{2}$ values of the order percent compared to those coming from the quarks and antiquarks.

The strength of virtual graviton exchange to the cross section contributions is characterised by the ratio $\lambda / M_{S}^{4}$. The coupling $\lambda$ depends on the full theory and is expected to be of order unity. Also the sign of interference with the Standard Model particles is a priori not known. Therefore the coupling is set to $\lambda= \pm 1$, following the convention of [11]. The data analysis is similar to the procedures described above. Gravitational effects are searched for by fitting the differential cross section to the above formulae with $\lambda / M_{S}^{4}$ treated as free parameter. The result of $\lambda / M_{S}^{4}=3.3_{-3.3}^{+4.2}{ }_{-1.3}^{+0.4} \mathrm{TeV}^{-4}$, where the second error reflects the parton density variation, is compatible with the Standard Model expectation. Lower limits at 95\% CL on $M_{S}$ for positive and negative coupling are then derived from the change in $\chi^{2}$ with respect to the Standard Model fit, yielding

$$
\begin{array}{ll}
M_{S}>0.48 \mathrm{TeV} & \text { for } \lambda=+1, \\
M_{S}>0.72 \mathrm{TeV} & \text { for } \lambda=-1 .
\end{array}
$$

Possible effects of graviton exchange to the data, as given by the exclusion limits, are illustrated in figure 6 .

Similar investigations of virtual graviton effects in $e^{+} e^{-}$annihilation into fermion and boson pairs provide comparable limits [23]. From the corresponding reaction of quark pair production scales of $M_{S}$ lower than $0.5-0.65 \mathrm{TeV}$ can be excluded. Combining all reactions that lead to two-fermion final states limits approaching $1 \mathrm{TeV}$ can be set.

\section{Conclusions}

Neutral current deep inelastic cross section measurements are analysed to search for new phenomena mediated through $(\bar{e} e)(\bar{q} q)$ contact interactions. No significant signal for compositeness, a quark form factor and virtual leptoquark or graviton exchange is found and the data are used to set limits which supersede and substantially improve former $\mathrm{H} 1$ results [2].

Limits on $(e q)$ compositeness are derived within a model independent analysis for scenarios involving one or more chiral couplings. The lower bounds on the scale parameters $\Lambda^{ \pm}$range between $1.3 \mathrm{TeV}$ and $5.5 \mathrm{TeV}$ for a coupling strength $g=\sqrt{4 \pi}$, depending on the chiral structure of the model.

A different approach to substructures is the concept of form factors. Such an analysis yields an upper limit of the size of the light up and down quarks of $R_{q}<1.7 \cdot 10^{-16} \mathrm{~cm}$ assuming a pointlike lepton. 
A study of virtual leptoquark exchange yields lower limits on the ratio $M_{L Q} / \lambda$ which for all types (except one) exceed the collider center of mass energy and approach $1 \mathrm{TeV}$ for vector leptoquarks with couplings to up quarks. These measurements complement and extend the direct leptoquark searches at HERA to high masses $M_{L Q}>\sqrt{s}$.

In a search for possible effects of low scale quantum gravity with gravitons coupling to Standard Model particles and propagating into extra spatial dimensions, lower limits on the effective Planck scale $M_{S}$ of $0.48 \mathrm{TeV}$ and $0.72 \mathrm{TeV}$ for positive and negative coupling, respectively, are found.

Acknowledgements. We are grateful to the HERA machine group whose outstanding efforts have made and continue to make this experiment possible. We thank the engineers and technicians for their work in constructing and now maintaining the $\mathrm{H} 1$ detector, our funding agencies for financial support, the DESY technical staff for continual assistance, and the DESY directorate for the hospitality extended to the non-DESY members of the collaboration. We gratefully acknowledge valuable discussions with G.F. Giudice.

\section{References}

[1] H1 collaboration, C. Adloff et al., DESY 99-107 [hep-ex/9908059, accepted by Eur. Phys. J. C.

[2] H1 collaboration, S. Aid et al., Phys. Lett. B 353 (1995) 578.

[3] H.-L. Lai et al., Eur. Phys. J. C 12 (2000) 375 and Phys. Rev. D 55 (1997) 1280.

[4] A.D. Martin et al., hep-ph/9907231, Eur. Phys. J. C 4 (1998) 463 and Phys. Lett. B 443 (1998) 301.

[5] M. Glück, E. Reya and A. Vogt, Z. Phys. C 67 (1995) 433.

[6] M. Botje, DESY 99-038 [hep-ph/9912439].

[7] E.J. Eichten, K.D. Lane and M.E. Peskin, Phys. Rev. Lett. 50 (1983) 811;

R. Rückl, Phys. Lett. B 129 (1983) 363 and Nucl. Phys. B 234 (1984) 91.

[8] P. Haberl, F. Schrempp and H.-U. Martyn, Proc. Workshop 'Physics at HERA', eds. W. Buchmüller and G. Ingelman, DESY, Hamburg (1991), vol. 2, p. 1133.

[9] N. Arkani-Hamed, S. Dimopolous and G. Dvali, Phys. Lett. B 429 (1998) 263 and Phys. Rev. D 59 (1999) 086004

[10] J.C. Long, H.W. Chan and J.C. Price, Nucl. Phys. B 539 (1999) 23.

[11] G.F. Giudice, R. Rattazzi and J.D. Wells, Nucl. Phys. B 544 (1999) 3;

G.F. Giudice, private communication: a statistical factor of 2 should be added to eq. (77). 
[12] OPAL collaboration, G. Abbiendi et al., Eur. Phys. J. C 6 (1999) 1 and CERN-EP/99-097 [hep-ex/9908008], submitted to Eur. Phys. J. C;

ALEPH collaboration, R. Barate et al., Eur. Phys. J. C 12 (2000) 183 and ALEPH CONF 99-013;

DELPHI collaboration, P. Abreu et al., Eur. Phys. J. C 11 (1999) 383;

L3 collaboration, M. Acciarri et al., Phys. Lett. B 433 (1998) 163.

[13] ZEUS collaboration, J. Breitweg et al., DESY 99-058 [hep-ex/9905039].

[14] CDF collaboration, F. Abe et al., Phys. Rev. Lett. 79 (1997) 2192;

D0 collaboration, B. Abbott et al., Phys. Rev. Lett. 82 (1999) 4769.

[15] A. Deandrea, Phys. Lett. B 409 (1997) 277.

[16] G. Köpp, D. Schaile, M. Spira and P.M. Zerwas, Z. Phys. C 65 (1995) 545.

[17] see reviews by T. Kinoshita and D.R. Yennie, H.-U. Martyn, and R.S. van Dyck Jr. in 'Quantum Electrodynamics', ed. T. Kinoshita, World Scientific (1990).

[18] J. Kalinowski, R. Rückl, H Spiesberger and P.M. Zerwas, Z. Phys. C 74 (1997) 595.

[19] H1 collaboration, C. Adloff et al., Eur. Phys. J. C 11 (1999) 447.

[20] K. Cheung, Phys. Lett. B 460 (1999) 383.

[21] P. Mathews, S. Raychaudhuri and K. Sridhar, Phys. Lett. B 455 (1999) 115.

[22] T. Rizzo, Phys. Rev. D 59 (1999) 115010.

[23] OPAL collaboration, G. Abbiendi et al., CERN-EP/99-097 [hep-ex/9908008], submitted to Eur. Phys. J. C;

L3 collaboration, M. Acciarri et al., Phys. Lett. B 464 (1999) 135 and Phys. Lett. B 470 (1999) 281;

ALEPH collaboration, ALEPH CONF 99-027. 

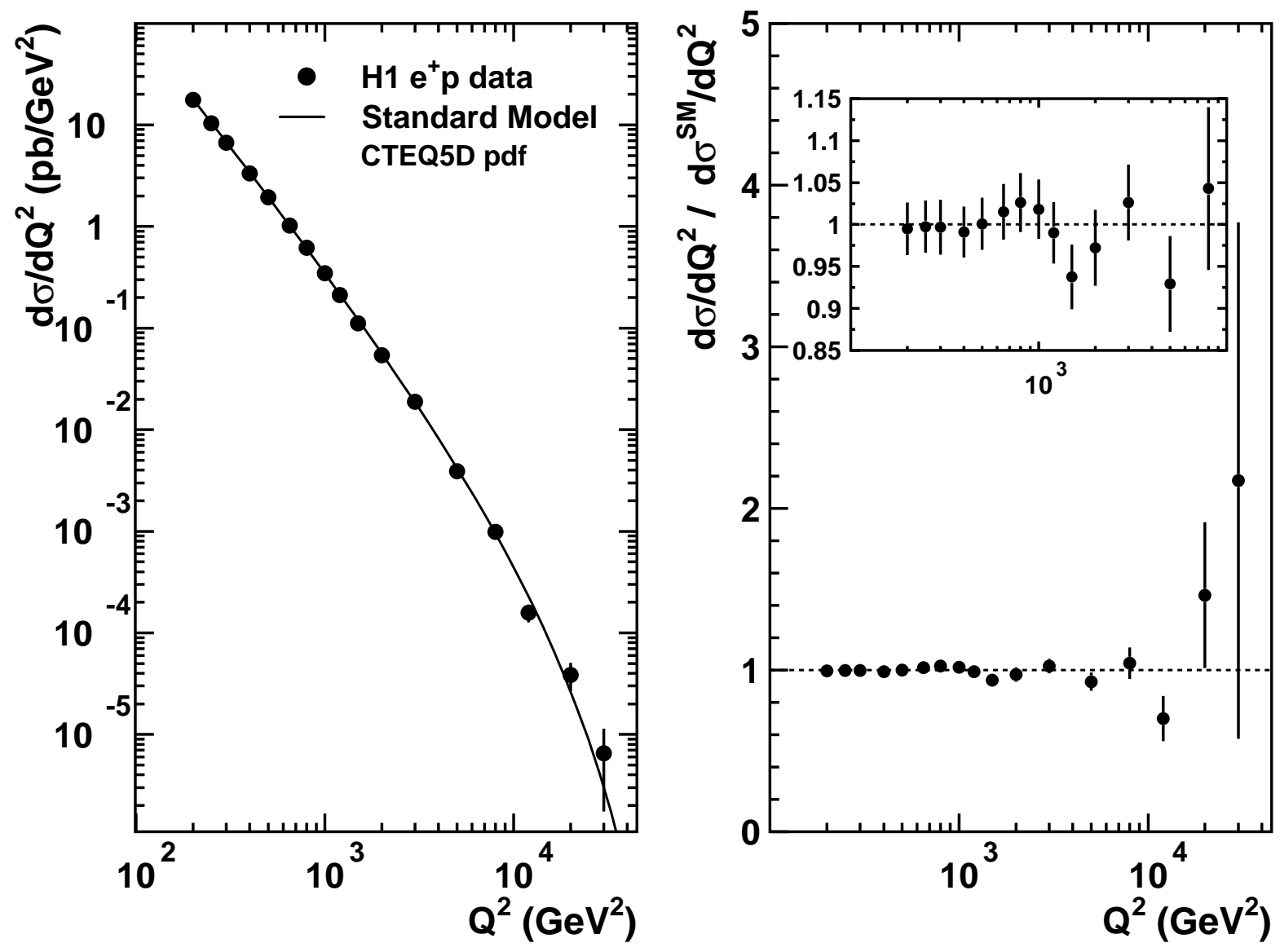

Figure 1: Differential NC cross section $\mathrm{d} \sigma\left(e^{+} p \rightarrow e^{+} X\right) / \mathrm{d} Q^{2}$. H1 data (•) are compared with the Standard Model expectation (-) using CTEQ5D parton distributions. The errors represent statistics and uncorrelated experimental systematics. The overall normalisation uncertainty is $1.5 \%$. 


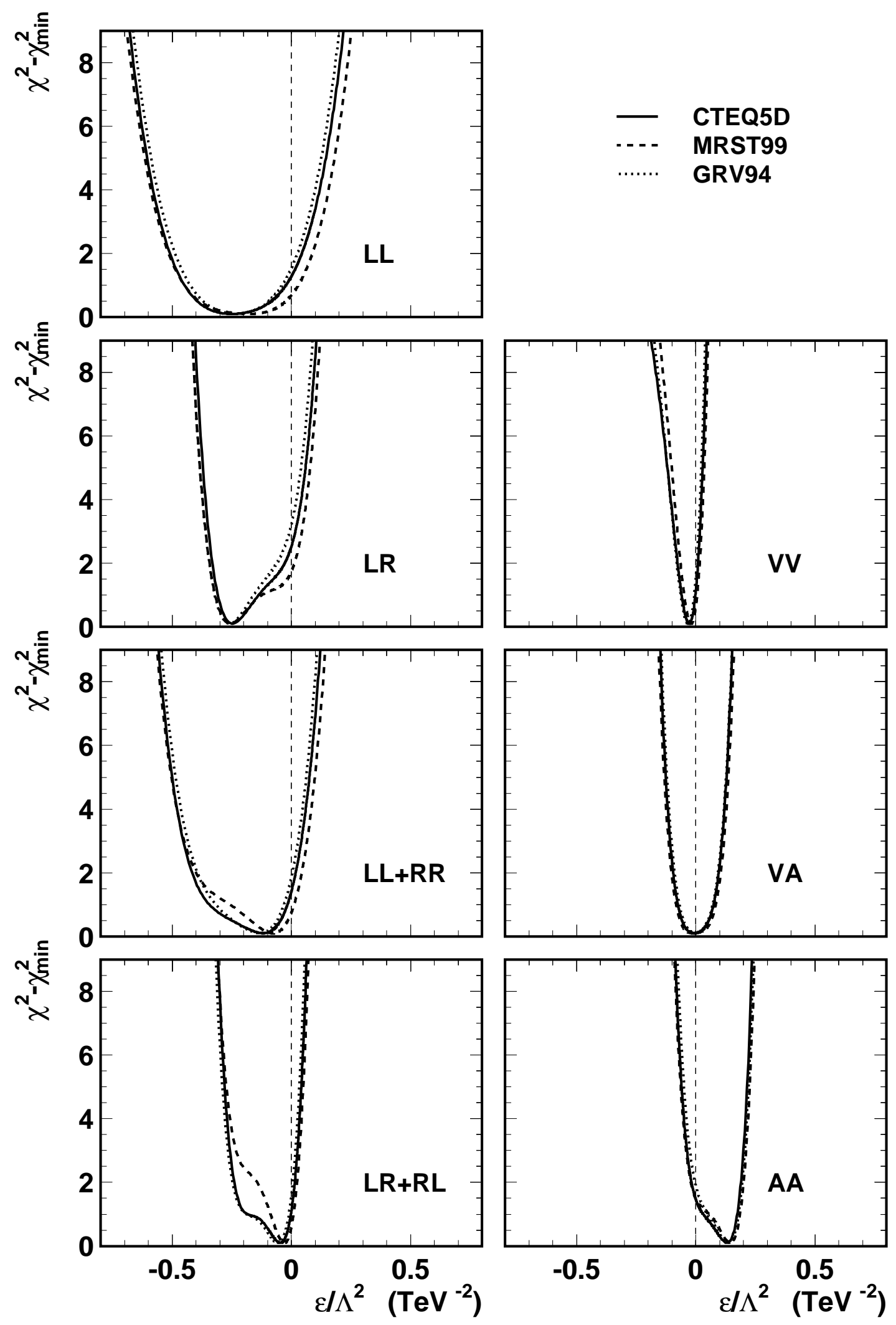

Figure 2: Distributions of $\chi^{2}-\chi_{\min }^{2}$ versus $\epsilon / \Lambda^{2}$ from fits to various compositeness models using CTEQ5D, MRST 99 and GRV 94 parton distributions and including full error propagation. 


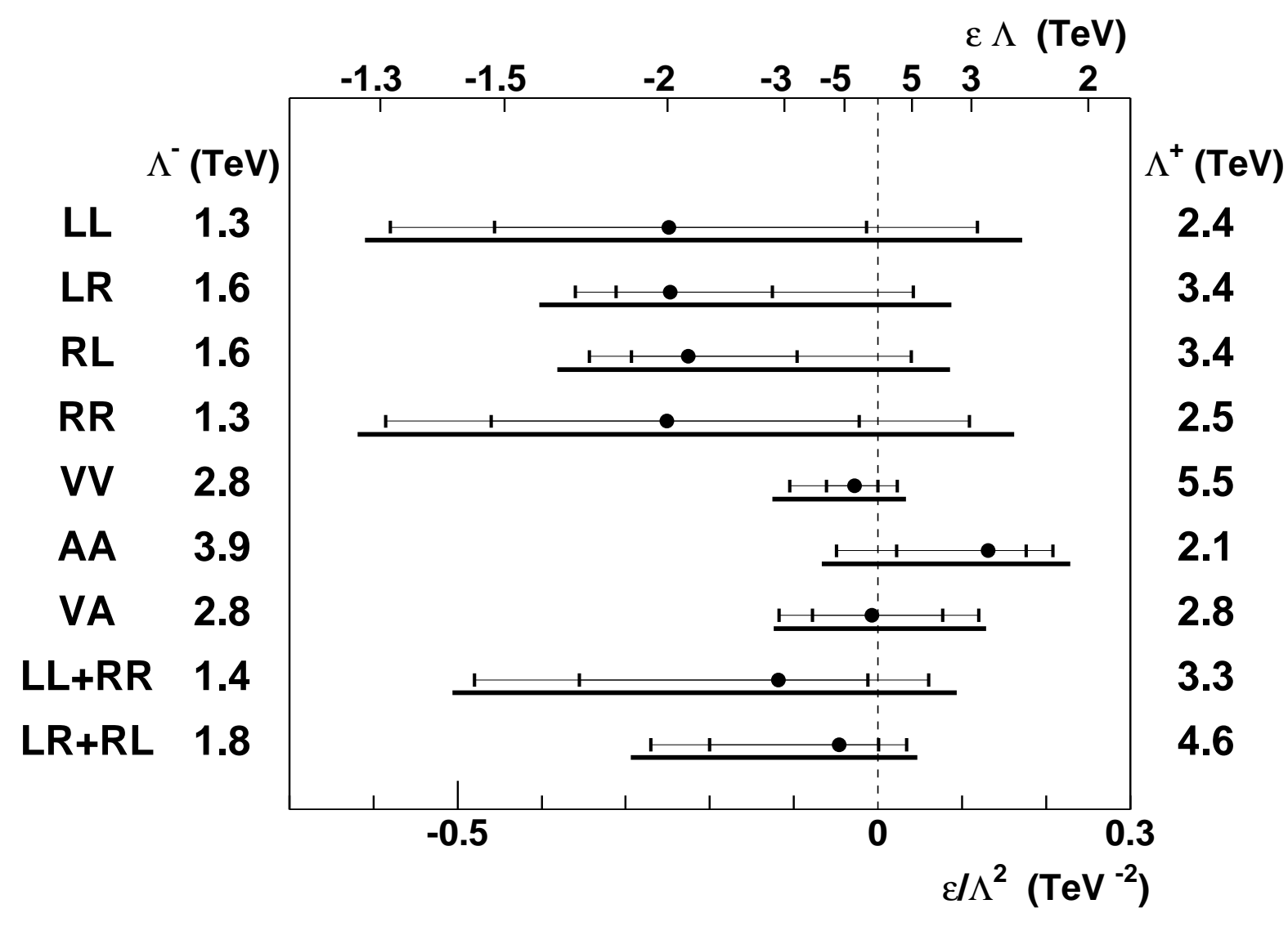

Figure 3: Analysis results of the parameter $\epsilon / \Lambda^{2}$ for various compositeness models. The thick horizontal bars indicate the limits on $\Lambda^{+}$and $\Lambda^{-}$including parton distribution uncertainties; values outside these regions are excluded at 95\% confidence level. The corresponding thin horizontal bars show the fit results for $\epsilon / \Lambda^{2}$ using CTEQ5D parton distributions; inner and outer error bars represent one and two standard deviations respectively. The scale for $\epsilon \Lambda$ is shown for convenience. 


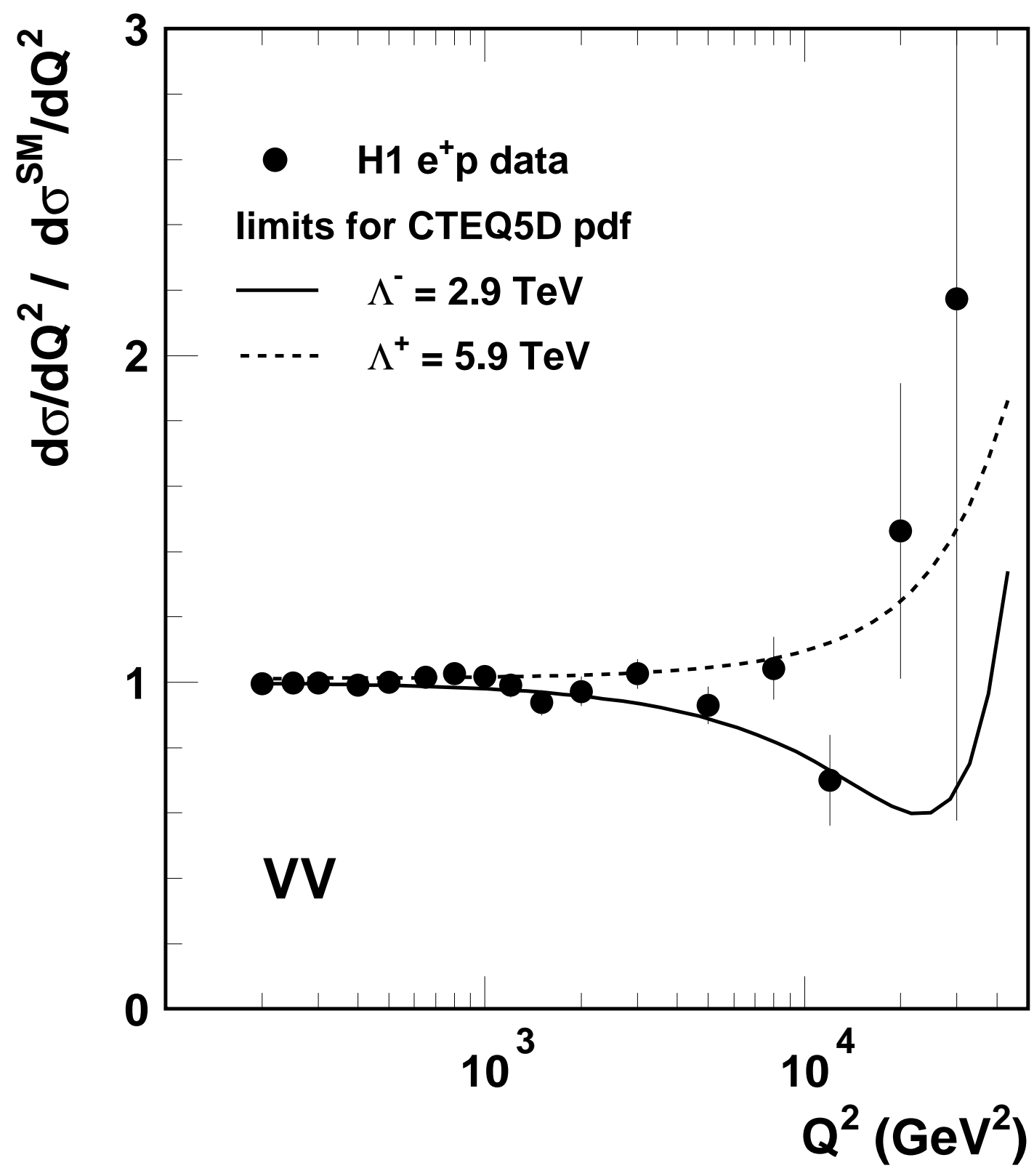

Figure 4: NC cross section $\mathrm{d} \sigma / \mathrm{d} Q^{2}$ normalised to the Standard Model expectation using CTEQ5D parton distributions. H1 data $(\bullet)$ are compared with fits to the VV model corresponding to $95 \% \mathrm{CL}$ exclusion limits of $\Lambda^{+}(--)$and $\Lambda^{-}(-)$. The errors represent statistics and uncorrelated experimental systematics. The overall normalisation uncertainty is $1.5 \%$. 


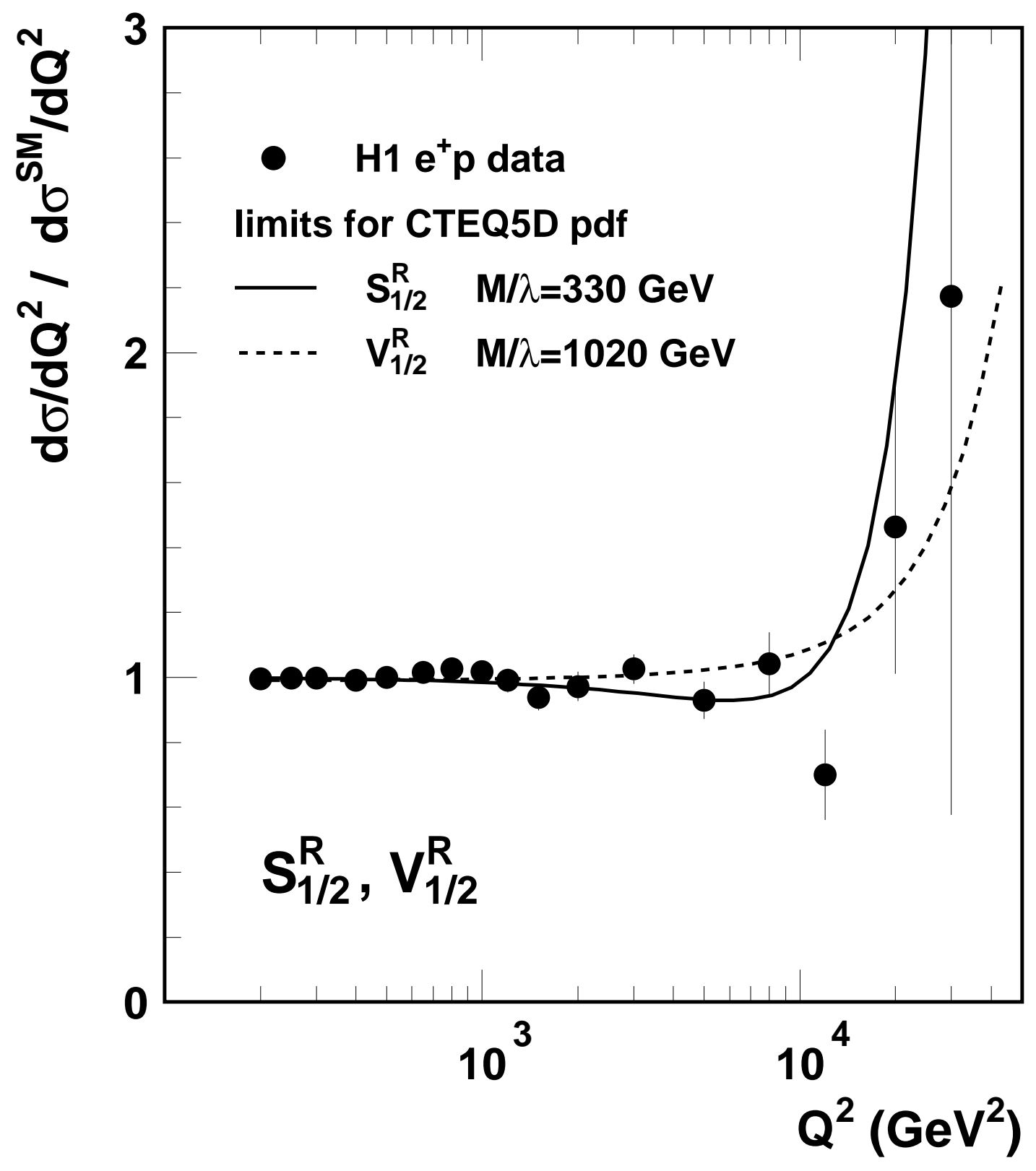

Figure 5: NC cross section $\mathrm{d} \sigma / \mathrm{d} Q^{2}$ normalised to the Standard Model expectation using CTEQ5D parton distributions. H1 data $(\bullet)$ are compared with 95\% CL exclusion limits of the leptoquarks $S_{1 / 2}^{R}(-)$ and $V_{1 / 2}^{R}(--)$. The errors represent statistics and uncorrelated experimental systematics. The overall normalisation uncertainty is $1.5 \%$. 


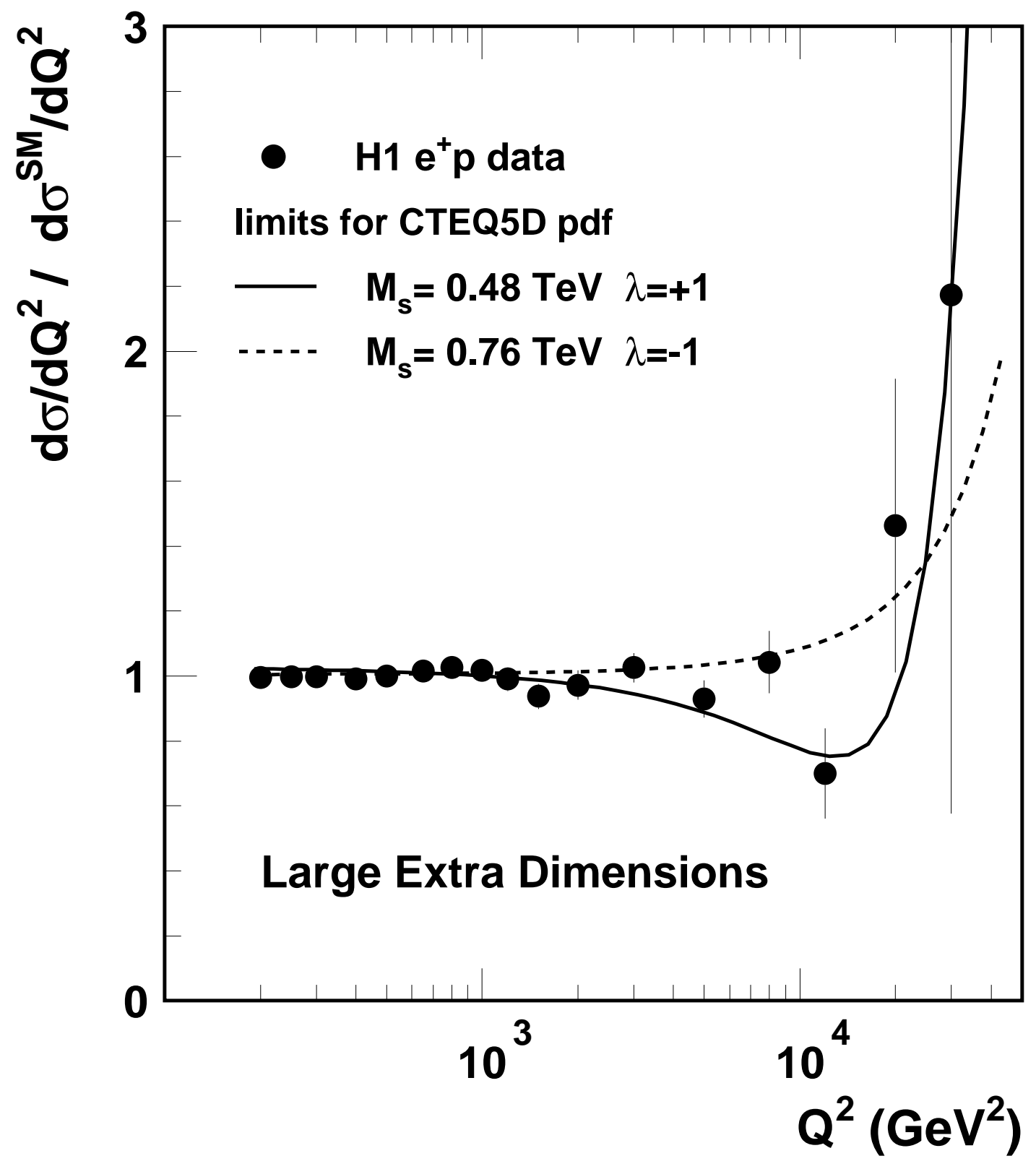

Figure 6: NC cross section $\mathrm{d} \sigma / \mathrm{d} Q^{2}$ normalised to the Standard Model expectation using CTEQ5D parton distributions. H1 data $(\bullet)$ are compared to the effect of graviton exchange given by the lower limits (95\% confidence level) on the scale $M_{S}$ for positive $(\lambda=+1$, full curve) and negative ( $\lambda=-1$, dashed curve) coupling. The errors represent statistics and uncorrelated experimental systematics. The overall normalisation uncertainty is $1.5 \%$. 